\title{
REPERCUSIONES PERINATALES TRAS VIOLENCIA DE GÉNERO EN LA MUJER EMBARAZADA. UNA REVISIÓN BIBLIOGRÁFICA ACTUALIZADA*
}

\author{
Marta de-Gracia-de-Gregorio ${ }^{1}$ \\ Correo electrónico: marta2189@ hotmail.com
}

1. Psicóloga Interna Residente, Servicio de Psiquiatría, Hospital Universitari de Bellvitge (L'Hospitalet de Llobregat, Barcelona).

Recepción: 26/11/2018 Aceptación: 18/01/2019

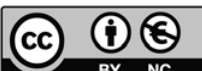

* Este trabajo ha sido presentado como comunicación oral en el III Congreso Internacional Género, Ética y Cuidado «Visibilizando la violencia hacia las mujeres en el embarazo» (2018). 


\section{RESUMEN}

\section{Introducción}

El embarazo no constituye un factor protector contra la violencia de género. Por contra, es una etapa crítica en la que se incrementa la vulnerabilidad de la mujer y donde la violencia puede aparecer, aumentar y prolongarse.

\section{Objetivos}

Revisión de la evidencia científica de los últimos años acerca de la relación entre violencia de género en la mujer embarazada y sus efectos sobre los resultados obstétricos y perinatales.

\section{Método}

Se ha realizado una revisión bibliográfica en las bases de datos online Pubmed, Tripdatabase y Cochrane de los últimos cinco años. Las palabras clave utilizadas para la búsqueda han sido las siguientes: intimate partner violence, domestic violence, pregnancy, premature, low birth weight, birth outcomes.

\section{Resultados}

La evidencia científica ha estudiado los efectos de la violencia de género sobre cuatro variables: parto prematuro (PP), bajo peso al nacer (BPN), restricción del crecimiento intrauterino (RCIU) y pequeño para la edad gestacional (PEG).

\section{Conclusiones}

Debido a las consecuencias sobre la salud materna y fetal causadas por la violencia de género, los estudios defienden la necesidad de establecer exploraciones de rutina en cada trimestre de gestación por parte de los sanitarios que están en contacto con la mujer embarazada. Así será más probable detectar una posible situación de riesgo, evitando daños y muertes en mujeres y bebés.

Palabras clave: violencia de género; mujer embarazada; consecuencias perinatales; prematuridad; bajo peso al nacer. 


\section{ABSTRACT}

\section{Introduction}

Pregnancy is not a protective factor against gender violence. On the contrary, it is a critical stage in which the vulnerability of women grows, contributing to the appearance, continuation and even the increase of violence.

\section{Objectives}

Review of the scientific evidence of recent years about the relationship between gender violence in pregnant women and its effects on obstetric and perinatal outcomes.

\section{Method}

A bibliographic review was made in Pubmed, Tripdatabase and Cochrane online databases, covering the last five years. The keywords used in the search were: intimate partner violence, domestic violence, pregnancy, premature, low birth weight, birth outcomes.

\section{Results}

Scientific evidence has studied the effects of gender violence on four variables: premature birth (PP), low birth weight (LBW), intrauterine growth restriction (IUGR), and small for the gestational age (SGA).

\section{Conclusions}

Due to the consequences on maternal and fetal health caused by gender-based violence, the studies defend the need to establish routine examinations in each trimester of pregnancy by health workers who are in contact with pregnant women. In this way, it will be more likely to detect a possible risk situation and thus avoid harm and the death of women and their babies.

Keywords: gender violence; pregnant woman; perinatal consequences; prematurity; low birth weight. 


\section{INTRODUCCIÓN}

La violencia contra la mujer es definida como «cualquier acto de violencia basada en género que produce o puede producir daños o sufrimientos físicos, sexuales o mentales en la mujer, incluidas las amenazas de tales actos, la coerción o la privación arbitraria de la libertad, tanto en la vida pública como privada» (Asambelea General de Naciones Unidas, 1993).

La violencia de género es una preocupación social que sufren las mujeres de todos los países del mundo. Es un problema que no solo pone en peligro la salud, la dignidad y la supervivencia de la mujer y la de sus hijos e hijas, sino que también afecta a sus aspiraciones y a la capacidad de control sobre sus vidas. Además, la violencia de género contribuye a la morbilidad física y psicológica y a la mortalidad de las mujeres entre 15 y 44 años en distintas culturas (Fischbach y Herbert, 1997).

Antiguamente, la violencia contra la mujer era considerada un problema del ámbito privado. Era un hecho cotidiano que únicamente concernía a los miembros de la familia. Por lo tanto, era un suceso normalizado, en que se justificaba el uso de la fuerza para mantener el orden y el control dentro de la intimidad del hogar. En definitiva, era un hecho invisible a ojos de la sociedad. Hasta los años 70, la violencia hacia la mujer en el ámbito doméstico no era reconocida generalmente. A partir de entonces, desde los movimientos feministas, se comenzó a demostrar la frecuencia de violencia en el ámbito familiar y sus efectos sobre las mujeres maltratadas (Lundy y Grossman, 2001). Según Walker (1999), aunque actualmente los datos sobre la ocurrencia de la violencia de género son abundantes, todavía es difícil descubrir muchas situaciones de maltrato, sobre todo cuando hablamos de maltrato psicológico.

En nuestro país, se sabe que alrededor de un $50 \%$ de las mujeres han sufrido alguna vez actos violentos en sus relaciones de pareja, y que un $25 \%$ ha padecido o padece una situación de violencia permanente (Pérez del Campo, 1995). En los casos más graves, la violencia dentro de la pareja termina con la muerte. Según un estudio realizado en los Estados Unidos, el homicidio era la segunda causa de muerte de las adolescentes de 15 a 18 años, y el $78 \%$ de las víctimas de homicidio comprendidas en el estudio habían sido matadas por un conocido o por su pareja (Organización de Naciones Unidas, 2006).

En cuanto al maltrato psicológico por parte de la pareja, también se trata de un problema importante. Aproximadamente el $35 \%$ de las mujeres denuncia ese tipo de 
abuso por parte de su pareja (O’Leary, 1999). Además, las mujeres suelen mostrar resultados psicológicos negativos mucho tiempo después del maltrato.

Se calcula que entre el $22 \%$ y el $35 \%$ de mujeres que acude a urgencias lo hace por violencia doméstica (Abbott et al., 1995). La violencia doméstica supone la causa más frecuente de lesiones en la mujer, más incluso que los accidentes de tráfico y los robos (Zubizarreta et al., 1994). Los estudios realizados muestran que entre un $37 \%$ y un $64 \%$ de las mujeres que han sufrido malos tratos por su pareja presenta lesiones físicas (Römkens, 1997). Por tanto, constituye un hecho que también implica a los profesionales sanitarios que han de atender a estas mujeres cuando acuden a los servicios de salud. Por otro lado, experimentar violencia de género supone una situación de estrés crónico, el cual está asociado a problemas de salud mental (Coyne y Downey, 1991). Si esta situación se perpetúa en el tiempo, se desarrollan una serie de síntomas y trastornos crónicos, entre los más prevalentes el Trastorno de Estrés Postraumático (TEPT). En un estudio de Echeburúa et al. (1997), observaron que un $55 \%$ de la muestra total del estudio presentaba sintomatología de estrés postraumático.

Respecto a la época perinatal, como apunta Távara-Orozco (2007), el embarazo no constituye un factor protector contra la violencia de género. Por contra, es una etapa crítica en la que incrementa la vulnerabilidad de la mujer y donde la violencia puede aparecer, aumentar y prolongarse. Daniel (2003) afirma que cada vez existe más evidencia de que, durante el embarazo y la maternidad, la mujer experimenta los primeros episodios de violencia o aumenta la frecuencia y severidad de los mismos. Según Peterson et al. (1998), la violencia que aparece alrededor del embarazo es la violencia física, sexual o psicológica, o las amenazas de violencia física o sexual infligida a una mujer antes del embarazo, durante el embarazo o en el puerperio o una combinación de estos períodos.

Son innegables las consecuencias sobre la salud física y psicológica de la mujer tras sufrir violencia por parte de su pareja o expareja. No obstante, en el caso de que la mujer esté embarazada, el bebé que está por nacer también sufre las consecuencias de esta violencia.

El objetivo de este trabajo es realizar una revisión de la evidencia científica de los últimos años acerca de la relación entre violencia de género en la mujer embarazada y sus efectos sobre los resultados obstétricos y perinatales. 


\section{MATERIAL Y MÉTODOS}

El diseño empleado en este estudio es el de una revisión bibliográfica. Se realizó una búsqueda exhaustiva en tres bases de datos en el primer trimestre de 2018. Las bases de datos consultadas son: PUBMED, TRIPDATABASE y COCHRANE.

Los descriptores empleados en inglés están incluidos en el Mesh: intimate partner violence, domestic violence, pregnancy, premature, low birth weight, birth outcomes. Se utilizó como enlace los operadores AND y OR. Los criterios de inclusión y exclusión tenidos en cuenta en la selección de los artículos y su posterior análisis se muestran en la Tabla 1.

Tabla 1. Criterios de inclusión y exclusión en la revisión de artículos

\begin{tabular}{|l|l|}
\multicolumn{1}{|c|}{ iterios de inclusión } & \multicolumn{1}{c|}{ Criterios de exclusión } \\
\hline Relacionado con los objetivos & No relacionado con los objetivos \\
\hline Publicaciones < 5 años & Publicaciones más antiguas de 5 años \\
\hline Idioma: inglés & Otros idiomas \\
\hline $\begin{array}{l}\text { Tipos de estudios: control aleatorizado, } \\
\text { revisiones sistemáticas y metaanálisis }\end{array}$ & \\
\hline
\end{tabular}

Así mismo, en la Figura 1 se muestra el flujo de información a través de las diferentes fases de la revisión. 
Figura 1. Flujo de información a través de las diferentes fases de la revisión

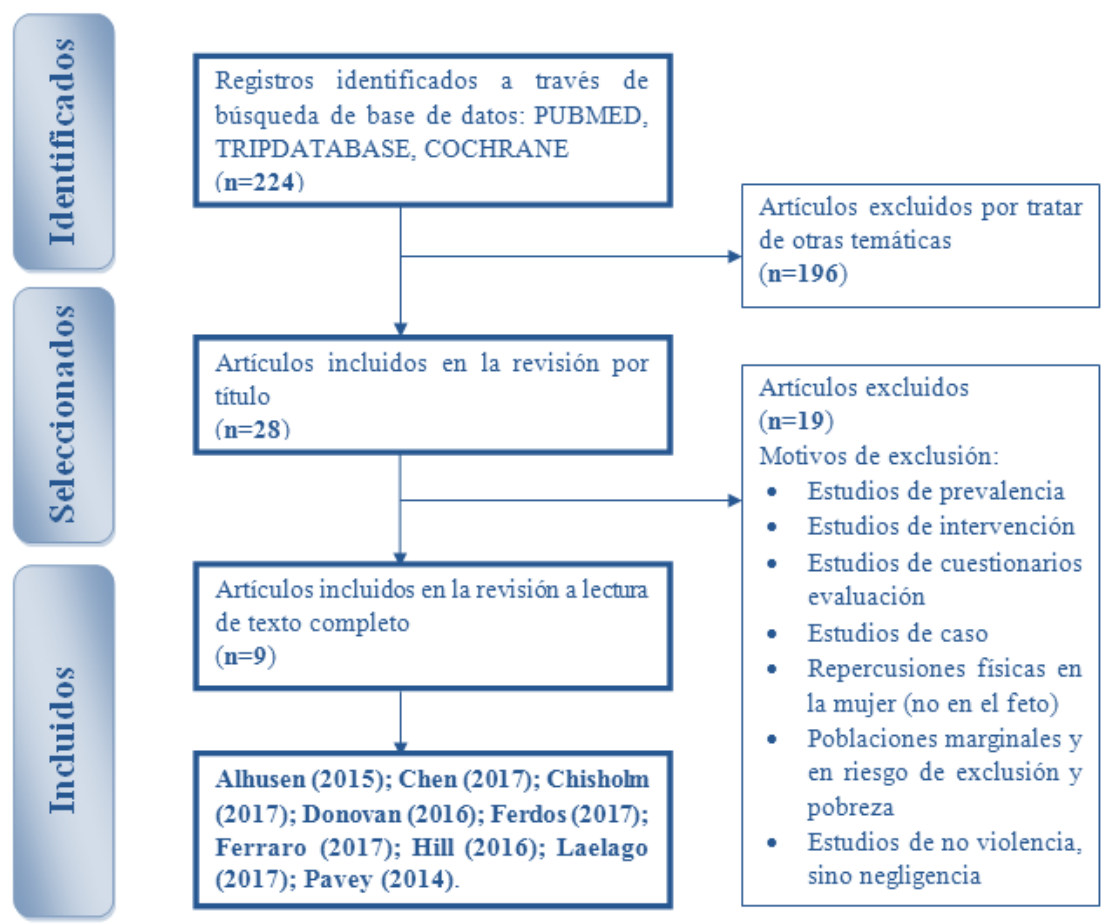

De la identificación inicial, se excluyeron aquellos artículos que trataban otras temáticas a partir de un primer análisis del título. De los artículos seleccionados por título, se realizó una lectura completa del texto, excluyendo aquellos que no se ajustaban al objetivo de este trabajo (por ejemplo, cuando se trataba de estudios de prevalencia de violencia de género, estudios de intervención psicológica, estudios de casos, estudios que exploraban las consecuencias físicas en la mujer, etc.). Tras la valoración de la adecuación de los trabajos, nueve artículos cumplieron los criterios de inclusión. De los artículos incluidos, se realizó una lectura crítica y la elaboración de los resultados. Finalmente, se elaboró una tabla donde se clasificaron teniendo en cuenta las siguientes variables: autoría y año de publicación, tipo de diseño, objetivos, resultados y conclusiones (Tabla 2).

\section{RESULTADOS}

La evidencia científica ha estudiado los efectos de la violencia de género sobre cuatro variables: parto prematuro (PP), bajo peso al nacer (BPN), restricción del crecimiento intrauterino (RCIU) y pequeño para la edad gestacional (PEG). La información obtenida en los distintos artículos se ha resumido en la Tabla 2. 


\begin{tabular}{|c|c|c|c|c|}
\hline $\begin{array}{l}\text { Autoría y } \\
\text { año }\end{array}$ & $\begin{array}{l}\text { Tipo de } \\
\text { diseño }\end{array}$ & Objetivos & Resultados & Conclusiones \\
\hline $\begin{array}{l}\text { Chen et al. } \\
\text { (2017) }\end{array}$ & $\begin{array}{l}\text { Estudio de } \\
\text { cohorte } \\
\text { retrospectivo }\end{array}$ & $\begin{array}{l}\text { Examinar el papel } \\
\text { de la violencia de } \\
\text { género en el } \\
\text { embarazo y su } \\
\text { asociación con } \\
\text { resultados adversos } \\
\text { en el nacimiento. }\end{array}$ & $\begin{array}{l}\text { Hay una asociación positiva entre violencia de } \\
\text { género en el embarazo y pobres resultados de } \\
\text { nacimiento. } \\
\text { En la población estudiada, una quinta parte de } \\
\text { las víctimas tuvieron partos prematuros y bebés } \\
\text { con bajo peso al nacer. Casi una cuarta parte de } \\
\text { los bebés de las víctimas recibieron cuidados } \\
\text { intensivos neonatales. Las tres medidas de } \\
\text { resultado analizadas están clínicamente } \\
\text { relacionadas. }\end{array}$ & $\begin{array}{l}\text { Se necesitan más estrategias de educación } \\
\text { y promoción de la salud que lleguen a } \\
\text { todas las mujeres embarazadas. Las } \\
\text { barreras en los sistemas sanitarios son: } \\
\text { falta de protocolos, tiempo limitado, falta } \\
\text { de recursos. } \\
\text { Se necesita más investigación para } \\
\text { desarrollar una intervención para mujeres } \\
\text { víctimas de violencia de género. }\end{array}$ \\
\hline $\begin{array}{l}\text { Chisholm, } \\
\text { Bullock y } \\
\text { Ferguson } \\
\text { (2017) }\end{array}$ & $\begin{array}{l}\text { Estudio de } \\
\text { revisión }\end{array}$ & No descrito & $\begin{array}{l}\text { La violencia de género se ha asociado con } \\
\text { resultados adversos del embarazo como son el } \\
\text { nacimiento prematuro, bajo peso al nacer y } \\
\text { pequeño para la edad gestacional. } \\
\text { Además, estos recién nacidos están en mayor } \\
\text { riesgo de desarrollar problemas del } \\
\text { comportamiento. } \\
\text { Los niños expuestos a violencia de género } \\
\text { tienen un mayor riesgo de daño fisiológico, } \\
\text { social y problemas emocionales y de } \\
\text { comportamiento, incluidos los trastornos del } \\
\text { estado de ánimo, ansiedad, trastorno de estrés } \\
\text { postraumático, abuso de sustancias y problemas } \\
\text { escolares. }\end{array}$ & $\begin{array}{l}\text { La estrategia ideal sería la prevención } \\
\text { primaria. En relación a la violencia de } \\
\text { género, se trataría de intervenir antes de } \\
\text { que aparezca la violencia. No obstante, se } \\
\text { destina menos del } 10 \% \text { de recursos a la } \\
\text { prevención primaria. Para que ésta tenga } \\
\text { éxito, se debe atacar la raíz de la violencia } \\
\text { de género: la desigualdad sexual. }\end{array}$ \\
\hline
\end{tabular}




\begin{tabular}{|c|c|c|c|c|}
\hline $\begin{array}{l}\text { Ferdos et } \\
\text { al. }(2017)\end{array}$ & $\begin{array}{c}\text { Estudio } \\
\text { descriptivo }\end{array}$ & $\begin{array}{l}\text { Examinar la relación } \\
\text { entre la experiencia } \\
\text { de violencia de } \\
\text { género materna y el } \\
\text { bajo peso al nacer. }\end{array}$ & $\begin{array}{l}\text { Una de cada tres bebés nació con bajo peso. } \\
\text { El riesgo de producir un hijo con bajo peso era } \\
\text { aún mayor cuando una mujer experimentaba } \\
\text { maltrato físico y sexual (cuatro veces mayor } \\
\text { que en una mujer que no sufría ningún tipo de } \\
\text { maltrato). }\end{array}$ & $\begin{array}{l}\text { Este estudio insta al gobierno a formular } \\
\text { políticas y aplicarlas adecuadamente para } \\
\text { evitar que las mujeres sean maltratadas por } \\
\text { sus parejas, para garantizar su salud y la de } \\
\text { sus hijos e hijas. } \\
\text { Se requiere, además, un examen adecuado } \\
\text { de detección de violencia de género para } \\
\text { proporcionar asesoramiento y apoyo social } \\
\text { a las mujeres. En este sentido, los servicios } \\
\text { de ginecología y obstetricia pueden ser } \\
\text { puntos clave de intervención para la } \\
\text { violencia de género. }\end{array}$ \\
\hline $\begin{array}{l}\text { Ferraro et } \\
\text { al. }(2017)\end{array}$ & $\begin{array}{l}\text { Estudio de } \\
\text { cohorte }\end{array}$ & $\begin{array}{l}\text { Determinar si existe } \\
\text { una asociación } \\
\text { mensurable entre } \\
\text { violencia doméstica } \\
\text { y trastornos } \\
\text { mentales, así como } \\
\text { con los resultados } \\
\text { en el nacimiento } \\
\text { (estado nutricional } \\
\text { del bebé y parto } \\
\text { prematuro). }\end{array}$ & $\begin{array}{l}\text { La violencia de género y los trastornos mentales } \\
\text { estuvieron altamente correlacionados en la } \\
\text { muestra de este estudio. } \\
\text { La combinación entre ansiedad y violencia } \\
\text { sexual se asoció con la duración del embarazo. }\end{array}$ & $\begin{array}{l}\text { A partir de este estudio, se puede concluir } \\
\text { que la violencia física y sexual, junto con } \\
\text { el trastorno de ansiedad, tienen un efecto } \\
\text { negativo sobre las variables de nacimiento } \\
\text { (peso al nacer, longitud y pequeño para la } \\
\text { edad gestacional). } \\
\text { La violencia doméstica tiene un efecto } \\
\text { perjudicial sobre los resultados del } \\
\text { nacimiento y es extremadamente } \\
\text { prevalente en áreas urbanas con pocos } \\
\text { recursos. }\end{array}$ \\
\hline
\end{tabular}




\begin{tabular}{|c|c|c|c|c|}
\hline $\begin{array}{l}\text { Laelago et } \\
\text { al. (2017) }\end{array}$ & $\begin{array}{l}\text { Estudio } \\
\text { transversal }\end{array}$ & $\begin{array}{l}\text { Examinar la } \\
\text { asociación entre } \\
\text { violencia de género } \\
\text { durante el embarazo y } \\
\text { resultados adversos } \\
\text { en el nacimiento. }\end{array}$ & $\begin{array}{l}\text { Hay una asociación entre violencia de género y bajo } \\
\text { peso al nacer del bebé. } \\
\text { La violencia de género no se asoció con muerte fetal, } \\
\text { nacimiento prematuro y puntuación Apgar menor a } 7 \text { a } \\
\text { los } 5 \text { minutos de nacimiento. }\end{array}$ & $\begin{array}{l}\text { La violencia de género se asocia } \\
\text { con un bajo peso al nacer del } \\
\text { bebé. } \\
\text { Hay dos posibles orígenes en } \\
\text { estos efectos adversos: } \\
\text { - Vía directa: a través de } \\
\text { agresión física directamente } \\
\text { en el abdomen. } \\
\text { - Vía indirecta: estrés materno } \\
\quad \text { elevado y deficiente nutrición. } \\
\text { Los servicios de salud deben } \\
\text { desarrollar programas para } \\
\text { detectar, tratar y hacer } \\
\text { seguimiento a las mujeres } \\
\text { maltratadas. }\end{array}$ \\
\hline $\begin{array}{l}\text { Donovan } \\
\text { et al. } \\
(\mathbf{2 0 1 6})\end{array}$ & $\begin{array}{l}\text { Revisión } \\
\text { sistemática y } \\
\text { metaanálisis }\end{array}$ & $\begin{array}{l}\text { Evaluar la relación } \\
\text { entre violencia de } \\
\text { género en el } \\
\text { embarazo y riesgo de } \\
\text { parto prematuro, bajo } \\
\text { peso al nacer y bebés } \\
\text { pequeños para la edad } \\
\text { gestacional. }\end{array}$ & $\begin{array}{l}\text { La violencia de género fue asociada de forma } \\
\text { significativa con parto prematuro y bajo peso al nacer. } \\
\text { La asociación con la variable pequeño para la edad } \\
\text { gestacional fue menos pronunciada, aunque hubo menos } \\
\text { estudios disponibles para el metaanálisis. }\end{array}$ & $\begin{array}{l}\text { Las mujeres que sufren violencia } \\
\text { de género durante el embarazo } \\
\text { tienen mayor riesgo de parto } \\
\text { prematuro y bebé con bajo peso al } \\
\text { nacer. } \\
\text { Se necesitan más estudios para } \\
\text { examinar la relación entre } \\
\text { violencia de género y la variable } \\
\text { pequeño para la edad gestacional. }\end{array}$ \\
\hline
\end{tabular}




\begin{tabular}{|c|c|c|c|c|}
\hline $\begin{array}{l}\text { Hill et al. } \\
(2016)\end{array}$ & $\begin{array}{l}\text { Revisión } \\
\text { sistemática y } \\
\text { metaanálisis }\end{array}$ & $\begin{array}{l}\text { Explorar la } \\
\text { asociación entre } \\
\text { violencia de género } \\
\text { en el embarazo y los } \\
\text { resultados adversos } \\
\text { en los nacimientos. }\end{array}$ & $\begin{array}{l}\text { La violencia de género durante el embarazo se asoció } \\
\text { con bajo peso al nacer y parto prematuro. No obstante, } \\
\text { no se encontró asociación estadísticamente significativa } \\
\text { con la restricción del crecimiento intrauterino ni para } \\
\text { bebé pequeño para la edad gestacional. Hay una relación } \\
\text { dosis-respuesta: cuanto mayor es la frecuencia de la } \\
\text { violencia durante el embarazo, mayores son las } \\
\text { probabilidades de parto prematuro y bajo peso al nacer. }\end{array}$ & $\begin{array}{l}\text { La evidencia científica muestra la } \\
\text { necesidad de incluir servicios } \\
\text { contra la violencia a las mujeres } \\
\text { en entornos de salud sexual y } \\
\text { reproductiva, particularmente en } \\
\text { el cuidado prenatal. } \\
\text { Estos servicios pueden ser puntos } \\
\text { de contacto importantes para } \\
\text { identificar la violencia de género } \\
\text { y vincular a las mujeres con los } \\
\text { servicios necesarios. }\end{array}$ \\
\hline $\begin{array}{l}\text { Alhusen et } \\
\text { al. (2015) }\end{array}$ & $\begin{array}{l}\text { Estudio de } \\
\text { revisión }\end{array}$ & $\begin{array}{l}\text { Describir los efectos } \\
\text { de la violencia de } \\
\text { género en la salud } \\
\text { materna y los } \\
\text { resultados neonatales } \\
\text { adversos. }\end{array}$ & $\begin{array}{l}\text { Hay evidencia en la literatura de la relación entre } \\
\text { violencia de género en el embarazo y aumento de la tasa } \\
\text { de parto prematuro y bajo peso al nacer. } \\
\text { Menos estudiada es la relación con pequeños para la } \\
\text { edad gestacional. La investigación que examina la } \\
\text { relación con esta variable ha arrojado resultados mixtos. } \\
\text { Hay asociación clínicamente significativa entre } \\
\text { violencia de género y riesgo de muerte perinatal. En un } \\
\text { estudio se mostró un riesgo hasta ocho mayor de muerte } \\
\text { gestacional y casi seis veces mayor de muerte neonatal. } \\
\text { Puede haber dos vías posibles para estas consecuencias: } \\
\text { - El traumatismo directamente sobre el abdomen o } \\
\text { violencia sexual. Esta situación puede aumentar los } \\
\text { mecanismos de afrontamiento desadaptativos en la } \\
\text { mujer: inadecuada o deficiente nutrición maternal, } \\
\text { aislamiento, acceso limitado a los controles } \\
\text { prenatales, consumo de tóxicos. }\end{array}$ & $\begin{array}{l}\text { Es importante, por parte de los } \\
\text { sanitarios, la evaluación e } \\
\text { intervención de la violencia de } \\
\text { género durante el embarazo. } \\
\text { Los esfuerzos para prevenir } \\
\text { eficazmente la iniciación de } \\
\text { violencia de género deberían } \\
\text { centrarse en las relaciones } \\
\text { saludables durante toda la vida, } \\
\text { con énfasis en los niños y los } \\
\text { jóvenes. }\end{array}$ \\
\hline
\end{tabular}




\begin{tabular}{|c|c|c|c|c|}
\hline & & & $\begin{array}{l}\text { El estrés psicológico puede afectar indirectamente, } \\
\text { exacerbando condiciones preexistentes como la } \\
\text { hipertensión y la diabetes gestacional, o puede } \\
\text { conducir a complicaciones del embarazo como la } \\
\text { preeclampsia. }\end{array}$ & \\
\hline $\begin{array}{l}\text { Pavey et } \\
\text { al. (2014) }\end{array}$ & $\begin{array}{l}\text { Estudio } \\
\text { retrospectivo }\end{array}$ & $\begin{array}{l}\text { Determinar el efecto } \\
\text { de la violencia } \\
\text { infligida por la pareja } \\
\text { en los resultados del } \\
\text { nacimiento y la } \\
\text { hospitalización de los } \\
\text { bebés. }\end{array}$ & $\begin{array}{l}\text { Los bebés nacidos de mujeres maltratadas tuvieron más } \\
\text { probabilidades de nacimiento prematuro, bajo peso al } \\
\text { nacer, diagnóstico de una enfermedad respiratoria, } \\
\text { hospitalización neonatal prolongada y reingresos. } \\
\text { Los bebés expuestos a violencia de género tuvieron un } \\
31 \% \text { más de probabilidades de experimentar alguna } \\
\text { consecuencia adversa. }\end{array}$ & $\begin{array}{l}\text { Los bebés expuestos a violencia } \\
\text { de género pueden requerir una } \\
\text { mayor utilización de los servicios } \\
\text { de salud, que podría extenderse a } \\
\text { la infancia y años posteriores. } \\
\text { Este estudio confirma las } \\
\text { asociaciones entre violencia de } \\
\text { género en el embarazo y } \\
\text { prematuridad y bajo peso al nacer, } \\
\text { así como la necesidad de } \\
\text { reingresos hospitalarios de los } \\
\text { bebés. }\end{array}$ \\
\hline
\end{tabular}




\section{Parto prematuro}

El nacimiento prematuro es definido médicamente como el parto ocurrido antes de 37 semanas de gestación, contadas desde el primer día de la última menstruación (Chen et al., 2017).

En la revisión de Donovan et al. (2016), treinta estudios informaron sobre la asociación entre PP y violencia de género, mostrando que existe casi el doble de probabilidades de que se produzca un nacimiento prematuro entre aquellas mujeres víctimas de violencia durante el embarazo, en comparación con aquellas mujeres que no estuvieron expuestas a dicha violencia.

Todos los estudios revisados han mostrado asociación estadísticamente significativa entre violencia de género y riesgo de parto prematuro, a excepción del estudio de Laelago et al. (2017).

\section{Bajo peso al nacer}

El bajo peso al nacer se define como aquel peso que está por debajo de un límite definido en cualquier edad gestacional (Ferdos et al., 2017).

En el estudio de Chen et al. (2017), una quinta parte de las víctimas tuvieron bebés con bajo peso al nacer.

Asimismo, en el estudio de Ferdos et al. (2017), uno de cada tres bebés nació con bajo peso al nacer. El riesgo de tener un hijo con bajo peso era aún mayor cuando una mujer experimentaba maltrato físico y sexual (cuatro veces más que una mujer que no sufría ningún tipo de maltrato).

Además, hay una relación dosis-respuesta: cuanto mayor es la frecuencia de la violencia durante el embarazo, mayores son las probabilidades de parto prematuro y de bajo peso al nacer (Hill et al., 2016).

\section{Pequeño para la edad gestacional y restricción del crecimiento intrauterino}

Pequeño para la edad gestacional se define como aquel peso que está por debajo del percentil 10 para la edad gestacional. Muchos de estos bebés están afectados por la llamada «restricción del crecimiento intrauterino», que ocurre cuando el feto no recibe 
los nutrientes y el oxígeno necesarios para el crecimiento y desarrollo adecuados de los órganos y tejidos (Hill et al., 2016).

Mientras que las variables de parto prematuro y bajo peso al nacer han mostrado relación significativa en la mayoría de los estudios, no sucede así para las variables de pequeño para la edad gestacional y de restricción del crecimiento intrauterino. Estas dos variables solo han sido revisadas en dos estudios, y ninguno de los dos han obtenido una asociación estadísticamente significativa (Donovan et al., 2016; Hill et al., 2016).

\section{Vías etiológicas}

La relación entre violencia de género durante el embarazo y sus resultados perinatales adversos tienen dos posibles vías etiológicas:

- Vía directa: sufrir una agresión física en el abdomen o bien una agresión sexual durante el embarazo se ha asociado con daño placentario, contracciones uterinas, rotura de membranas e infecciones genitourinarias. Esto puede aumentar el riesgo de aborto espontáneo, parto prematuro, bajo peso al nacer y muerte fetal (Ferdos et al., 2017; Laelago et al., 2017; Alhusen et al., 2015).

- Vía indirecta: el estrés materno sufrido durante el maltrato puede activar el eje neuroendocrino, causando la liberación de cortisol y catecolaminas. Esto provoca la vasoconstricción en los vasos placentarios, lo que conduce a la hipoxia fetal y al trabajo de parto prematuro. Además, puede agravar problemas crónicos de salud (como diabetes, hipertensión, asma) en las que las mujeres embarazadas podrían estar en un nivel de riesgo más elevado. También puede aumentar conductas de riesgo no saludables en la mujer (como consumo de tabaco y alcohol) (Ferdos et al., 2017; Laelago et al., 2017; Alhusen et al., 2015).

\section{DISCUSIÓN}

Según el Plan Nacional de Sensibilización y Prevención de la Violencia de Género (Ministerio de Trabajo y Asuntos Sociales, 2008), la violencia ejercida contra las mujeres constituye en primera instancia un atentado contra su salud física y psicológica. De este modo, los profesionales sanitarios cobran un protagonismo muy relevante tanto en la detección del fenómeno como en la atención a las víctimas. Este Plan Nacional se construye en torno a dos parámetros de actuación: 
- La Prevención, distinguiendo los niveles de prevención primaria, secundaria y terciaria. Asumir estos tres niveles implica contar con recursos e instrumentos de socialización y de restauración de un plan de vida de las mujeres víctimas de violencia de género.

- La Sensibilización, dotando a la sociedad de herramientas necesarias para que sepan reconocer cuándo se inicia o cuando se está ante un proceso de violencia.

La presente revisión muestra la creciente base de pruebas de que la violencia de género en las mujeres, cuando están embarazadas, no solo tiene repercusiones sobre la mujer, sino también en el futuro bebé.

Según la evidencia científica, las consecuencias de eventos estresantes repercuten tanto en la duración del embarazo como en el tamaño corporal del recién nacido.

Estos hallazgos muestran la necesidad de incluir servicios para la mujer, en relación a la violencia de género, en los servicios de salud sexual y reproductiva, particularmente en el cuidado prenatal (Hill et al., 2016).

Los protocolos de identificación de violencia durante el embarazo pueden ayudar a detectarla y a intervenir con mayor rapidez y efectividad por parte de los servicios de salud para prevenir las complicaciones anteriormente mencionadas (Chen et al., 2017).

Para lograr estos objetivos, sería necesario impulsar un programa de sensibilización del personal de salud (médicos, enfermeras, trabajadores sociales y psicólogos) que entra en contacto cotidianamente con las mujeres maltratadas en los diferentes niveles de atención a la salud (Ferdos et al., 2017).

Una de las limitaciones del trabajo ha sido la escasa bibliografía que estudia las variables «pequeño para la edad gestacional»y «restricción del crecimiento intrauterino», encontrando únicamente dos estudios. No obstante, la mayoría de la bibliografía se centra en estudiar las variables «parto prematuro»y «bajo peso al nacer». Por ello, sería interesante que los estudios también se centraran en aportar evidencia sobre otras consecuencias perinatales.

\section{CONCLUSIONES}

Según los datos de la presente revisión, la violencia de género que sufren las mujeres embarazadas no solo repercute física y emocionalmente en las mujeres, sino también en 
el bebé. La literatura aporta datos suficientes de que la mujer en estado de gestación no está protegida de la violencia de género; al contrario, es una etapa en la que puede iniciarse o exacerbarse. Por lo tanto, se encuentra en un período especialmente vulnerable, y más teniendo en cuenta que en el embarazo es importante el autocuidado físico y emocional para procurar un correcto desarrollo del futuro bebé.

Teniendo en cuenta este hecho, sería importante y necesario incluir protocolos de actuación en los servicios de salud. Los controles prenatales que la mujer embarazada ha de realizarse $\mathrm{y}$, por tanto, la vinculación con los servicios de salud pueden ser una oportunidad para realizar una entrevista de diagnóstico de violencia de género. De esta manera, si se identifican episodios de maltrato y se ponen en marcha los recursos para brindar apoyo a la mujer, se estará protegiendo al mismo tiempo a dos generaciones. 


\section{BIBLIOGRAFÍA}

Аввотт, J. et al. Domestic violence against women: Incidence and prevalence in an emergency department population. En: Journal of the American Medical Association. 1995, vol. 273, pp. 1763-7.

ALHUSEN, J. L. et al. Intimate partner violence during pregnancy: maternal and neonatal outcomes. En: Journal of women's health. 2015, vol. 24, núm. 1, pp. 100-6.

Asamblea General de Naciones Unidas. Declaración sobre la eliminación de la violencia contra la mujer [en línea]. Asamblea General de las Naciones Unidas, 1993. [Consulta: 22 de noviembre de 2018]. Disponible en: 〈www.ohchr.org/sp/professionalinterest/pages/violenceagainstwomen.aspx $>$.

CHEN, P. H. et al. Birth outcomes in relation to intimate partner violence. En: Journal of the national medical association. 2017, vol. 109, núm. 4, pp. 238-45.

Chisholm, C. A.; Bullock, L.; Ferguson, J. Intimate partner violence and pregnancy: epidemiology and impact. En: American journal of obstetrics \& gynecology. 2017, vol. 217, núm. 2, pp. 141-4

Coyne, J. C.; Downey, G. Social factors and psychopathology: Stress, social support, and coping processes. En: Annual Review of Psychology. 1991, vol. 42, pp. 401-25.

DANIEL, K. Hitting home. Community Practitioner, 76, 12, 457-458.

DONOVAN B. M. et al. Intimate partner violence during pregnancy and the risk for adverse infant outcomes: a systematic review and meta-analysis. En: BJOG. 2003, vol. 123, pp. 1289-99.

ECHEBURÚA, E. Repercusiones psicopatológicas de la violencia doméstica: un estudio descriptivo. En: Revista de Psicopatología y Psicología Clínica. 1997, vol. 2, núm. 1, pp. 7-19.

Ferdos, J.; RAHMAN, M. M. Maternal experience of intimate partner violence and low birth weight of children: A hospital-based study in Bangladesh. En: Plos one. 2017, vol. 12, núm. 10 .

FERrARo, A. A. et al. The specific and combined role of domestic violence and mental health disorders during pregnancy on new-born health. En: BMC Pregnancy and Childbirth. 2017, vol. 17, núm. 1, pp. 257.

FISCHBACH, R. L.; HeBert, B. Domestic violence and mental health: correlates and conundrums within and across cultures. En: Social Science and Medicine. 1997, vol. 45, pp. 1161-76. 
HILL, A. et al. A systematic review and meta-analysis of intimate partner violence during pregnancy and selected birth outcomes. En: International Journal of Gynecology and Obstetrics. 2016, vol. 133, pp. 269-76

Laelago, T.; Belachew, T.; TAMRAT, M. Effect of intimate partner violence on birth outcomes. En: African health sciences. 2017, vol. 7, núm. 3.

LunDY, M.; GROSSMAN, S. Clinical research and practice with battered women. What we know, what we need to know. En: Trauma, Violence, \& Abuse. 2001, vol. 2, pp. 120 41.

Ministerio de Trabajo y Asuntos Sociales. Plan Nacional de Sensibilización y Prevención de la Violencia de Género [en línea]. Madrid: Ministerio de Trabajo y Asuntos Sociales, 2008. Disponible en:

<https://www.cop.es/GT/Plan_nacional_sensibilizacion_prevencion_violencia_gene ro.pdf>.

O’LeARY, K. D. Psychological abuse: A variable deserving critical attention in domestic violence. En: Violence and Victims. 1999, vol. 14, pp. 3-23.

ORGANIZACIÓN DE NACIONES UNIDAS. Estudio a fondo sobre todas las formas de violencia contra la mujer: Informe del Secretario General (Resolución de la Asamblea General 61/122 de 6 de julio de 2006) [en línea]. ONU, 2006. Disponible en: 〈www.un.org/es/comun/docs/?symbol=A/61/122/Add.1 $>$.

PAVEY, A. R. et al. Intimate partner violence increases adverse outcomes at birth and in early infancy. En: The journal of pediatrics, 165. 2014, vol. 5, pp. 1034-9.

PÉREZ DEL CAMPO, A. M. Una cuestión incomprendida. El maltrato a la mujer. Madrid: Horas y Horas, 1995.

PETERSON, R. et al. Key scientific issues for research on violence occurring around the time of pregnancy. Atlanta (Georgia): National Center for Chronic Disease Prevention and Health Promotion, 1998. Disponible en:

$<$ http://ia601702.us.archive.org/27/items/keyscientificiss00cent/keyscientificiss00ce nt.pdf>.

RÖMKENS, R. Prevalence of wife abuse in the Netherlands. Combining quantitative and qualitative methods in survey research. En: Journal of Interpersonal Violence. 1997, vol. 12, pp. 99-125.

TÁVARA-OROZCO, Luis et al. Repercusiones maternas y perinatales de la violencia basada en género. En: Revista Peruana de Ginecología y Obstetricia. 2007, vol. 53, núm. 1, pp. $10-17$ 
WALKER, L. Psychology and domestic around the world. En: American Psychologist. 1999, vol. 54, pp. 21-29.

Zubizarreta, I. et al. Consecuencias psicológicas del maltrato doméstico. En: Echeburúa, Enrique (ed.). Personalidades Violentas. Madrid: Pirámide, 1994, pp. 129-51. 